\title{
An Extension of Abraham-Minkowski Controversy to Extend Abraham-Minkowski Friendship: A Theoretical Study in Astrophysics
}

\author{
Md. Abdul Hakim \\ Department of Food Technology and Nutritional Science, Mawlana Bhashani Science and Technology University, Santosh, Tangail, \\ Bangladesh
}

Email address:

info.hakim.bd@gmail.com

\section{To cite this article:}

Md. Abdul Hakim. An Extension of Abraham-Minkowski Controversy to Extend Abraham-Minkowski Friendship: A Theoretical Study in Astrophysics. International Journal of Discrete Mathematics. Vol. 3, No. 1, 2018, pp. 28-31. doi: 10.11648/j.dmath.20180301.14

Received: March 19, 2018; Accepted: April 23, 2018; Published: May 14, 2018

\begin{abstract}
The Abraham-Minkowski controversy of light refracting to a system is a century-old milk and water at light refraction on a medium. The physicists are in long debate to pick and choose the Abraham or Minkowski forma at calculating momentum of light in a medium during reflection at dynamic environment in astrophysics. This study is designed to ride an anchor in view of mitigating these ongoing hot water in physical geospatialities. Results divulged Abraham-Minkowsky friendship on the equation (16) envelop can turn over a new leaf to the contrary of these Abraham-Minkowski controversy in light physics. Also, it can be a uniquely functional modeling to be used as $\rho=\eta \mathrm{E} / \mathrm{c}=\mathrm{E} / \eta \mathrm{c}$ dancing in worldwide confusion over the controversry.
\end{abstract}

Keywords: Abraham-Minkowski Controversy, Refraction, Abraham-Minkowsky Friendship, Refractive Index, Astrophysics, Theoretical Study

\section{Introduction}

Light is a moving particles generated from an electromagnetic radiation called photons $[1,2]$. The photon is the single particle of light having field vector of electric charge $[3,4]$. The photon is a massless particle occupying the momentum $[5,6]$. This photon is a natural agent stimuting the sight and making things around us visible [7]. This is usually refer to visible light to the human eye creating the sense of sight where visible light is defined as the light of wavelengths ranging 400 to $700 \mathrm{~nm}$, between the infrared and the ultraviolet rays $[8,9]$.

The photonic body of sunlight is the suprb over other manual light sources in living world [10]. This light is moving sometimes in wave forms and sometimes in straight lines maintaining the rules of reflection sometimes and refraction sometimes based on environmental geospatialities $[11,12]$. Refraction is the change of direction of wave propagation due to change in its trasmission medium in different settings [13]. Reflraction follows Snell's law as $\mathrm{v}_{1} \sin \theta_{2}=\mathrm{v}_{2} \sin \theta_{1} ; \theta_{1}$ is the angle of incidence, $\theta_{2}$ is the angle of refraction and $v_{1} / v_{2}$ is the phase velocities in the dual media in the system $[14,15]$. The momentum of the refracted rays is going to be on brown study now-a-days due to Abraham- Minkowski controversry in moderm physics [16, 17]. Therefore, this study is conducted in quest of a meddle with bid to escape from this controversy forming a modeling as a new leaf naming Abraham-Minkowsky friendship in worldwide astrophysics.

\section{Methodology}

This is a methodological review study in cross-sectional multivariate analysis using secondary data analysis method. Secondary data refers to data collected by some researchers in their studies and by some writers in their scientific books. This data analysis technique in mathematical and physical statistics is in galore use to attain the ultimate gaining in a new study. The data is collected from the speed of light, its mass, velocity, momentum and Snell's law in theoretical physics as well as Newtonian mechanics, refractive index, phase velocity, Minkowski and Abraham forma, Stephen Barnett postulates, Albert Einstein's mass-energy equivalence in astrophysics. These theoretical and thematic instruments are used in 
different mathematical calculations in applied physics. These instruments have then taken the turn on inventing AbrahamMinkowsky friendship in equational gesture aiming to overcome Abraham-Minkowski controversry at light photon reflection in modern engineering physics.

\section{Results and Discussions}

Momentum is the product of the mass and velocity of an object. It is a $3 \mathrm{D}$ vector quantity, possessing a magnitude and a direction [18-20].

Let, $\mathrm{m}$ is an object's mass, $\mathrm{v}$ is its velocity and $\rho$ is the momentum. We get the following equation in this fair play in Newtonian mechanics,

$$
\rho=m v
$$

Considering $\rho$ as the momentum of light, $m$ as its mass and $\mathrm{v}$ as the speed in vacuum, we get,

$$
\mathrm{v}=\mathrm{c}
$$

where,

$\mathrm{c}=$ speed of light in vacuum $\left(\approx 3 \times 10^{8} \mathrm{~ms}^{-1}\right)$ [21-23].

Putting the value of equation (2) into equation (1),

$$
\rho=\mathrm{mc}
$$

In 1908, Hermann Minkowski calculates the momentum $\rho$ of the refracted ray [24-27] as follows:

$$
\rho=\eta_{M} E / c
$$

where,

$\eta_{M}=$ refractive index of the medium stated in Minkowski's calculation,

$\mathrm{E}=$ energy of photon and

$\mathrm{c}=$ speed of light in vacuum.

Just a year later, in 1909, Max Abraham proposed the following formula [28-32] in this connection,

$$
\rho=\mathrm{E} / \eta_{\mathrm{A}} \mathrm{c}
$$

where,

$\eta_{\mathrm{A}}=$ refractive index of the medium stated in Abraham's calculation,

$\mathrm{E}=$ energy of photon and

$\mathrm{c}=$ speed of light in vacuum.

A 2010 study suggested by physicist Stephen Barnett propsed the correctness of both the Abraham's and the Minkowski's formulas on his one study in applied physics in measuring the momentum of the refracted ray [33]. The Barnett's study is on the laughing stock to some physicists in the worlwide theoretical mathematics and enegineering physics. A comperison between Abraham and the Minkowski momenta is prevailed till today to the physicists [34].

Let give up these cofusion rather than knowing the definition of refractive index in astrophysics. The refractive index is the dimentionless number applied to describe the light propagation nature through the medium and mathematically it is on the following dance [35-38],

$$
\eta=\mathrm{c} / \mathrm{v}
$$

where,

$\mathrm{c}=$ speed of light in vacuum and

$\mathrm{v}=$ phase velocity of light in the medium [39-41].

Let us have a look at a glance on mass-energy equivalence derived by world renowned physicist Albert Einstein as the energy $\mathrm{E}$ is on the following modeling [42-45],

$$
\mathrm{E}=\mathrm{mc}^{2}
$$

Investigating the equations (3) and (4), we get,

$$
\begin{gathered}
\eta_{M} E / c=m c \\
\text { Or, } \eta_{M} E=m c 2
\end{gathered}
$$

Again, Investigating the equations (3) and (5), we get,

$$
\begin{aligned}
\mathrm{E} / \eta_{\mathrm{A}} \mathrm{c} & =\mathrm{mc} \\
\text { Or, } \mathrm{E} & =\eta_{\mathrm{A}} \mathrm{mc}^{2}
\end{aligned}
$$

Now, dividing the equation (8) by the equation (7), we get,

$$
\begin{aligned}
\eta_{M} E / E & =\mathrm{mc}^{2} / \mathrm{mc}^{2} \\
\text { Or, } \eta_{M} & =1
\end{aligned}
$$

Again, dividing the equation (9) by the equation (7), we get,

$$
\begin{aligned}
\mathrm{E} / \mathrm{E} & =\eta_{\mathrm{A}} \mathrm{mc}^{2} / \mathrm{mc}^{2} \\
\text { Or, } \eta_{\mathrm{A}} & =1
\end{aligned}
$$

Investing the duo equations (11) and (12), we get,

$$
\eta_{\mathrm{M}}=\eta_{\mathrm{A}}=1
$$

investigating equation (6) and (12), we get,

$$
\eta_{\mathrm{M}}=\eta_{\mathrm{A}}=1=\eta
$$

Putting the value of equation (13) in equation (4) and (5), we get,

$$
\begin{gathered}
\rho=1 \times E / c=\eta E / c \\
\rho=E / 1 \times c=E / \eta c
\end{gathered}
$$

The overall investigation of equations (13) and (14) directs the following modeling,

$$
\rho=\eta \mathrm{E} / \mathrm{c}=\mathrm{E} / \eta \mathrm{c}
$$

The equation (16) can be a solvable bid to the peril prevaining on Abraham and Minkowsky controversy in an anew heiring to Abraham-Minkowsky friendship in astrophysics giving vent to the derived equation (16) in the study.

\section{Conclusions}

The battle between Minkowsky and Abraham formula is 
the greatest World War in astrophysics and in applied mathematics. The current study dynamics has shown a smooth route to deviate this war rendering the mathematical relation $\rho=\eta \mathrm{E} / \mathrm{c}=\mathrm{E} / \eta \mathrm{c}$ in equation (16). Future research should adopt this cozy modeling for taking intervention to understand the aptness of Minkowsky and Abraham formula in astrophysics and also in computation mathematics.

\section{Completing of Interests}

The author declares that he has no competing interests.

\section{Acknowledgements}

The author wish to thanks Dr Azizur Rahman, a Professor at School of Computing and Mathematics of Charles Sturt University, Wagga Wagga, New South Wales in Australia who shares me different philosophy on mathematical physics on the score of drafting this manuscript. The author is very greatful to the little angels, the learners at Hakim Shadow Education Approach (HakimSEA) at Karatia of Tangail in Bangladesh who keep me in the good book on writing this manuscript.

\section{References}

[1] Pal GK and Pal P (2001). Textbook of practical physiolofy ( $1^{\text {st }}$ ed.). Chennai: Orient Blackswan; p. 387.

[2] Moulder JE (2007). Static electric and magnetic fields and human health.

[3] Maxwell JC (1891). Treatise on electricity and magnetism. Dover Publications; p. 32-33.

[4] Ryder LH (1996). Quantum field theory ( $2^{\text {nd }}$ edn.). Cambridge University Press.

[5] Fedtke C, Mans F and Ho Arthur (2010). The entrance pupil of the human eye: a three-dimensional model as a function of viewing angle. Optics Express 18 (21): 22364-76.

[6] Rindler W (1991). Introduction to special relativity ( $2^{\text {nd }}$ edn.). Oxford Science Publications; p. 82-84.

[7] Assad MEH, Bani-Hani EH, Yousef B, Sedaghat and Tawalbeh M (2018). Simplified model for thermo and diffusiophoretic deposition in heat exchanger. JP J Heat Mass Transfer 15 (1): 1-13.

[8] Avison J (1999). The world of physics. Nelson Thornes; p. 460.

[9] Quandt BM, Scherer LJ, Boesel LF, Wolf M, Bona GL and Rossi RM (2015). Body monitoring and health supervision by means of optical fibers based sensing systems in mediacal textiles. Adv Healthc Mater 4 (3): 330-55.

[10] Lekner J (1987). Theory of reflection, of electromagnetic and particle waves. Springer.

[11] Chen R and Guinness RE (2014). Geospatial computing in mobile devices $\left(1^{\text {st }}\right.$ edn.). Norwood, MA: Artech House; p. 228 .
[12] Rahman A (2017). Estimating small area health-related characteristics of populations: a methodological review. Geospat Health 12: 3-14.

[13] Agrawal M (2010). Business data communications. John Wiley and Sons, Inc.; p. 37-43.

[14] Ward DW, Nelson KA and Webb KJ (2005). On the physical origins of the negative index of refraction. New J Phys 7: 213.

[15] Born and Wolf (1959). Principles of optics. New York, NY: Pergamon Press Inc.; p. 37.

[16] Wang Zhong-Yue, Wang Pin-Yu and Xu Yan-Rong (2011). Crutial experiment to resolve Abraham- Minkowski controversry. Optik 122 (22): 1994-96.

[17] Hakim MA (2017). Mathematical modeling of energy balancing for diet planning in nutritional physics. Int $J$ Nutr Diet 5 (1): 29-41.

[18] McGill and King (1995). Engineering mechanics, an introduction to dynamics ( $3^{\text {rd }}$ ed.). PWS Publishing Company.

[19] Serway RA and John WJJ (2012). Principles of physics: a calculus-based text $\left(5^{\text {th }}\right.$ ed.). Boston, MA: Brooks/Cole, Cengage Learning; p. 245.

[20] Griffiths DJ (2013). Introduction to electrodynamics ( $4^{\text {th }}$ ed.). Boston, Mass.: Pearson; p. 361.

[21] Penrose R (2004). The road to reality: a complete guide to the laws of the the universe. Vintage Books; p. 410-11.

[22] Mendelzson KS (2006). The story of c. Am J Phys 74 (11): 995-97.

[23] Chambers A (2004). Moderm vacuum physics. Boca Raton: CRC Press.

[24] Minkowski H (1908). Die Grundgleichung fur die elektromagne tischen Vorgange in betwegten Kopern. Nachrichten von der Gesellschaftcer Wissenschaften zu Gottingen, Mathematisch-Physikalische Klasse: 53-111.

[25] Bor Z, Osvay K, Racz B and Szabo C (1990). Group refractive index measurement by Michelson interferometer. Optics Communication 78 (2): 109-112.

[26] Dickson LE (1909). Review: geometric der Zahlen von Hermann Minkowski. Bull Amer Math Soc 21 (3): 131-32.

[27] Wilson EB (1915). Review: Gesammelte Abhandlungen von Herman Minkowski. Bull Amer Math Soc 21 (8): 409-12.

[28] Abraham M (1909). Zur Elektrodynamik betwegter Kopern. Rendiconti del Circolo Matematico oi Palermo 28 (1).

[29] Ciddor PE (1996). Refractive index of air: new equations for the visible and near infrared. Applied Optics 85 (9): 1566-73.

[30] Abraham M (1903). Prinzipien der Dynamik des Elektrons. Annalen der Physik 10: 105-79.

[31] Hakim MA (2018). Trending malnutritional escape velocity in nutritional dynamics. Food Sci Technol 6: in press.

[32] Fitzgerald R (2000). Phase-sensitive X-ray imaging. Phys Today 53 (7): 23.

[33] Barnett S (2010). Resolution of Abraham-Minkowski dilemma. Phys Rev Lett 104 (7): 070401. 
[34] Testa M (2016). A comperison between Abraham and the Minkowski momenta. J Modern Phys 7: 320-28.

[35] Kinsler LE (2000). Fundamentals of acoustics. John Wiley; p. 136.

[36] Newton I (1730). Optics: on a treatise of the reflections, refractions, infections and colours of light; p. 247.

[37] Brewster D (1815). On the structure of doubling refracting crystals. Philosophycal Magazine 45: 126.

[38] Hakim MA and Kamruzzaman A (2016). The dance of poverty and education for childhood nutritional victimization in Bangladesh. J Bilogic Environ Eng 1 (1): 6-9.

[39] Nemirovsky J, Rechtsman MC and Segev M (2012). Negative radiation pressure and negative effective refractive index via dielectric birefringence. Optics Express 20 (8): 8907-14.
[40] Brillouin L (1960). Wave propagation and group velocit, New York and London: Academic Press Inc.

[41] Einstein A (1916). Relativity: the special and general theory. New York: H. Holt and Company.

[42] Mehra J (2001). Albert Einstein's first paper, the golden age of physics, World Scientific.

[43] Rahman A and Hakim MA (2016). Measuring modified mass energy equivalence in nutritional epidemiology: a proposal to adapt the biophysical modeling approach. Int J Stat Med Res 5 (3): 219-223.

[44] Rahman A and Hakim MA. Modeling health status using the logarithmic biophysical modulator. J Public Health Epidemiol 2017; 9 (5): 145-150.

[45] Hakim MA (2017). Biophysical modeling of dietary energy in biochemical modeling. Eur J Biophys 5 (3): 57-61. 